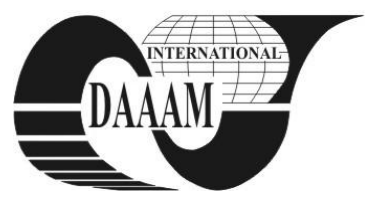

Annals of DAAAM for 2012 \& Proceedings of the 23rd International DAAAM Symposium, Volume 23, No.1, ISSN 2304-1382 ISBN 978-3-901509-91-9, CDROM version, Ed. B. Katalinic, Published by DAAAM International, Vienna, Austria, EU, 2012 Make Harmony between Technology and Nature, and Your Mind will Fly Free as a Bird Annals \& Proceedings of DAAAM International 2012

\title{
CONTRIBUTIONS TO THE MODELING OF A COMMUNICATION CHANNEL BY RBF
}

\author{
PIRLOAGA, M[arian] D[orin] \& MIHAILESCU, M[arius] I[ulian]
}

\begin{abstract}
This paper's aim is to model a communicationchannel, done at the level of a complex equalizer, using a transversal filter, $K$ ordered. Here are being studied the in and out signals, their noise influence. We propose the training of a RBFR network with a control less algorithm to determine the weightings on the interconnections of the exit layer. Finally, we draw some conclusions about the efficiency of equalizer towards the RNM implemented models.
\end{abstract}

Keywords: equalizer, communication channel, RBF neural network, interconnections, decision regions

\section{INTRODUCTION}

Communication channels have, in the most general case, phase and amplitude, linear and time variables characteristics, due to inherent nonlinearities, weather propagation conditions, extra environmental noises and thermal noise of electronic devices. Amplifiers that usually function close to the saturation point also introduce memory less nonlinearities, which, combined with the effects of the transmission and reception filters, becomes memory nonlinearities. Consequently, the transmitted signals are affected by different nonlinear distortions, attenuations, extra noise, intersymbol interference, interference with adjacent channels etc [1].

The reconstruction technique of transmitted symbols is the channel equalization. In case of a big leakage of the entry values, linear equalizers face the problem of numerical instability. Given the fact that, in general, communication channels are variable in time, equalizers must be adaptive, to follow in time the variations of the frequency answer of the channel $[3,6]$. In the case of nonlinear distortions, generated by time-variable channels, linear equalizers don`t have good performances. The only solution in case of nonlinear and severe distortions of the communication channels is the nonlinear equalizers. Approaching the problem of equalization as a classification problem, the neuronal networks can generate arbitrary regions of decision with great precision.

In radar, sonar and communication applications, ideal signals are usually contaminated with non-Gaussian noise. The radar performance can be degraded by impulsive noise interference such as environmental effects of atmospherics (lighting) and meteor train echoes. Lighting impulsive noise significantly reduces the signal detector performance about 25 percentage. Detection of known signals from noisy observations is an important area of statistical signal processing with direct applications in the communications fields. General properties of neural networks include robustness and fault tolerance of the computational elements due to the massive parallesim. [30]
Also, the adaptive neural networks that vary with time are able to change with slowly time-varying signals, improving the non-Gaussian signal detection performance. The neural networks are nonparametric, making no assumptions about the underlying densities, which may provide more robustness and capability for detecting signals generated by nonlinear and nonGaussian processes.

Linear models have been studied in statistics field for more then 200 years and the theory can be applied in RBF networks, which represent one single type of linear model.

\section{THE PROPOSED IDEA}

Equalizer contains a linear transversal filter $m$ ordered and a RBFR. Communication channel is modeled using a $k$ ordered transversal filter. Being given the vector of the signal from the exit of the communication channel $\boldsymbol{y}[n\rceil$, which is affected by additional complex noise $\boldsymbol{w}[n]$, can be Gaussian noise with dispersion. The signals $\boldsymbol{y}[n]$ and $\boldsymbol{w}[n]$ are considered uncorrelated. Real part $w R[n]$ and the imaginary $w I[n]$ of the noise are considered mutual dependent sequences [1]. The exit of the communication channel is applied to the entrance of the complex equalizer. The vector of the received signal applied to the entrance RBFR is the sequence:

$$
\boldsymbol{r}[n], \boldsymbol{r}[n]=[r[n] r[n-1] \ldots r[n-m+1]] T
$$

Because this implies $m$ terms of the entrance signal, will be considered NS possible combinations of the entrance sequence in the communication channel.

$$
\begin{aligned}
& N S=4 M+m-1 \text { which has the form } \boldsymbol{x}[n]= \\
& {[x[n] x[n-1] \ldots x[n-m-M+2]] T}
\end{aligned}
$$

The corresponding signal from the exit of the communication channel unaffected by noise is the vector:

$$
\boldsymbol{y}[n]=[y[n] y[n-1] \ldots y[n-m+1]] T,
$$

which also has NS states. The task of the equalizer is to reconstruct the sended signal as precisely as it can, by generating an estimate, based on the vector of the received signal $\boldsymbol{r}[n]$ and the delayed signal $x[n-d]$. The real part, respectively the imagined one of the complex signals 4-QAM is processed independently, in the same manner. The unlimited activation function of the hidden neurons is real and can be specified through the relation:

$$
\varphi=\varphi\left((\mathrm{r}[\mathrm{n}]-\mathrm{ci}[n])^{H} \mathrm{r}[\mathrm{n}]-\mathrm{ci}[\mathrm{n}] / \rho\right), 1 \leq \mathrm{i} \leq \mathrm{Nh}
$$




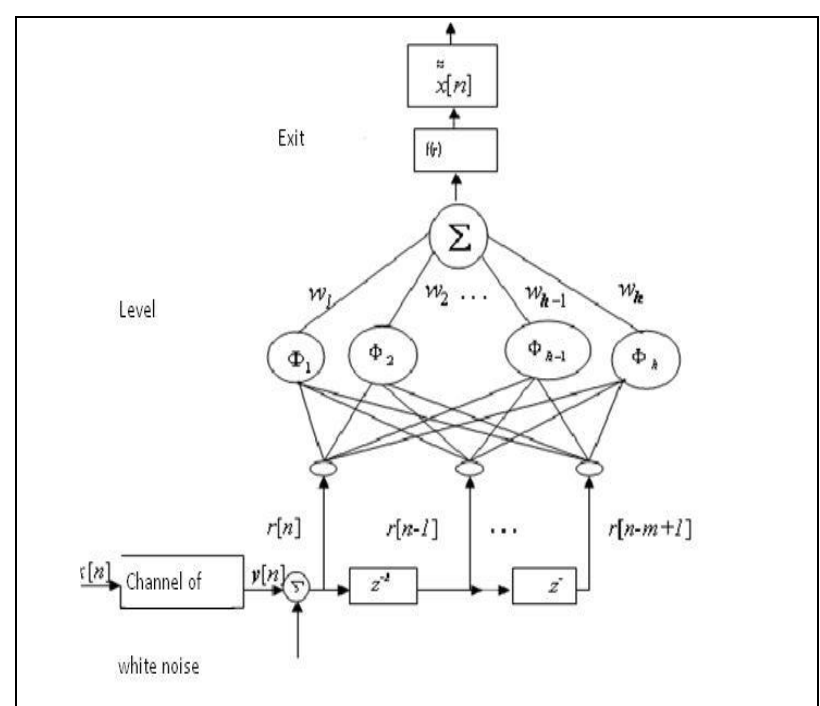

Fig 1. Block scheme of the equalizer

The operator $(\bullet) H=((\bullet) T)^{*}$ is $(\bullet) T$ the transposition operator and $(\bullet *$ is the conjugation complex operator. The exit layer of the RBFR has eight neurons, by two neurons for each of the possible classes of the signal 4QAM, one for the real part and the other for the imaginary part. The activation function of the exit neurons fRBF is linear and, considering the Gaussian activation function of the hidden neurons, it is given the relation:

$$
f R B F R(r)=\sum_{i=1}^{N h} w i e-\frac{\left(r[n]-c i[n]^{H}\right)}{\pi}
$$

where wi are the complex interconnection weightings towards the exit neuron.

To train the RBFR network is normally used a control less algorithm to determine the centers of the hidden layer and one with control to determine the weightings of the interconnections of the exit layer [10, 8, 34-7]. An example of algorithm with customary (usual) control is the algorithm of the least mean square error (LMS), which utilizes the following relation to determine the weightings of the interconnections towards the exit layer of a RBFR.

$$
\left.w_{i}[n+1]=w_{i}[\mathrm{n}]+\alpha e[n] \nsubseteq n\right]
$$

Where $\alpha$ is the learning constant and $e[n]$ is the complex error, determined with the relation:

$$
e[n]=x[n-d]-f_{R B F}(r)
$$

This algorithm minimizes the average of the square error:

$$
M S E=\frac{1}{N \cdot \sum_{i=1}^{n} e_{i}^{2}[n]}
$$

Other algorithms allow the simultaneous determination of all the parameters RBFR. An example is the algorithm proposed by Cha and Kassam [13] in the study that utilizes as error criteria a stochastic descendant gradient.
The Algorithm calculates the current gradient of the mean square error and modifies the parameters of the network towards its minimization. An algorithm with sequential learning for the training of a neuronal equalizer complex algorithm with complex minimal resource allocation network CMRAN, allows the adding and elimination of hidden neurons, so that it realizes an optimal structure for the given application. The initial network has no hidden neurons. Each time when at the entry is applied a pair of training data, formed of an entry - exit wished model, the network is build based on three growing models. The algorithm adds a hidden neuron or modifies the existing parameters of $\mathrm{RN}$ according to the training data. The CMRAN algorithm also incorporates a removal mechanism of the hidden neurons that don't significantly contribute to RN performance. High orders communication channels have a big number of states, so that they require RBFR structures with a big number of centers. CMRAN algorithm reduces yet the number of centers so that the implemented equalizer has a good performance, by means of a reduced complexity and so of a rapid working process.

\section{THE RESULTS OF IMPLEMENTATION}

The signals of 4-QAM were generated using an uniform distribution, the real part independently from the imaginary one. It was generated a complex white noise $w[n]$ with a Gaussian distribution, the real part independently from the imaginary one [1]. By choosing the order of the model of channel $k=3$ and the order of the linear filter and the order of the transversal linear filter from the entry of RBFR $m=1$, it is obtained the number of possible states of the signal from the channel exit, $N S=64$. For the exit status of RBFR were utilized eight neurons, by twos for each possible class of the signal 4-QAM, one for the real part and one for the imaginary part [20], [21], [22], [23], [24], [25], [26], [27], [28], [29], [30], [31], [32], [33]. The radius of radial activation functions, $\rho$, was chosen to have value 0.28 . To determine the centers of the equalizer RBFT were applied $N=1000$ entry sequences:

$$
x[n]=[x[n] x[n-1] x[n-2]
$$

The signals from the exit of communication channel, receipted signals affected by noise $\boldsymbol{r}[n]$, initial and final positions of the centers of RBFR center $c[n]$, in case of $\mathrm{RSZ}=13 \mathrm{~dB}$, after 100 iterations (Legend: '0` - wished states, '*'- entry noisy states, ' $\mathrm{X}$ ' - initial positions of the centers, ' $+`$ - final positions of the centers).

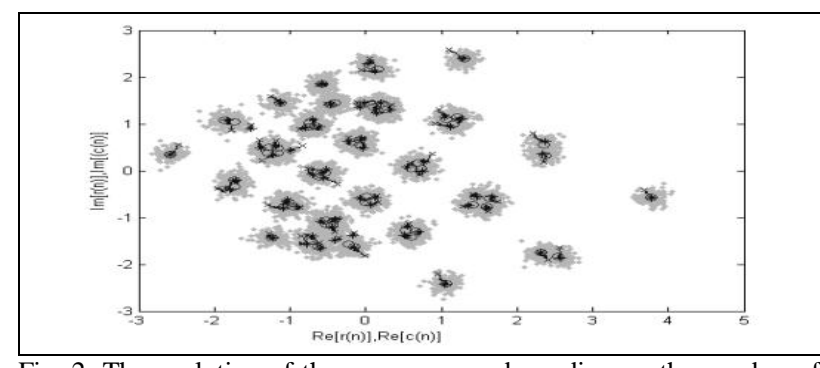

Fig. 2. The evolution of the square mean depending on the number of training periods for $\mathrm{RSZ}=5 \mathrm{~dB}$ and complex nonlinear channel of $m=1$ and $d=1$ 


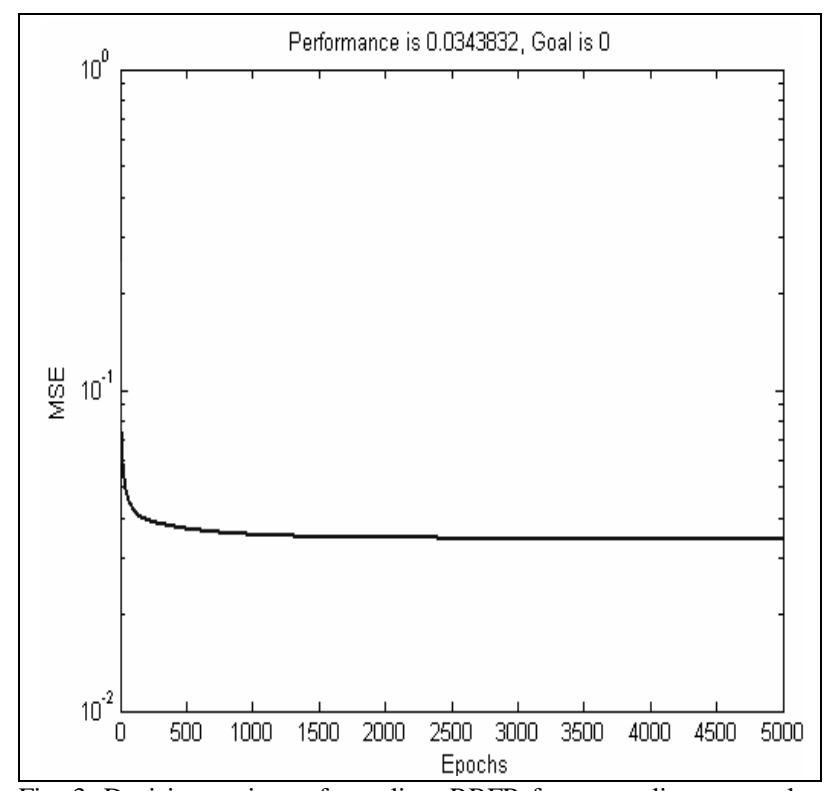

Fig. 3. Decision regions of equalizer RBFR for an nonlinear complex channel in case: $\mathrm{RSZ}=5 \mathrm{~dB}, m=1$ and a delay $d=1$

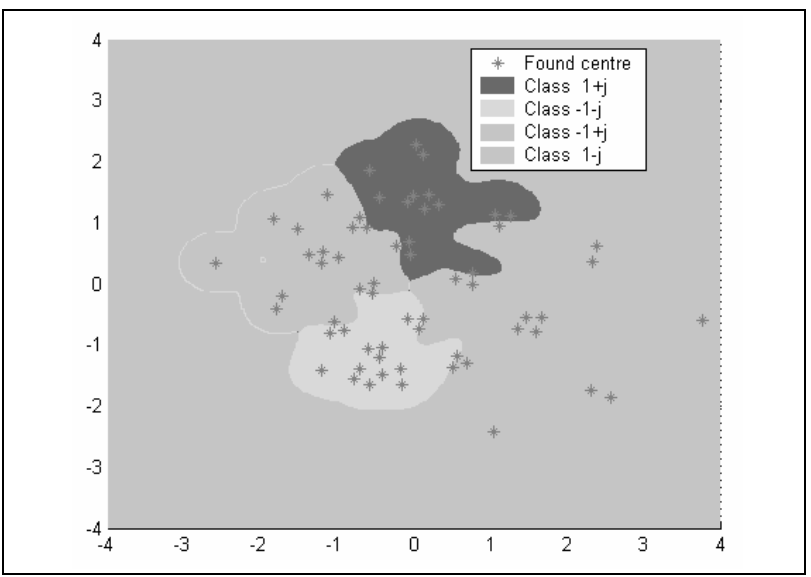

Fig. 4. Analyze of the signals at exit of equalizer

The signals from the exit of the communication channel, noise affected signals $\boldsymbol{r}[n]$, initial and final position of centers RBFR networks $c[n]$, in case of a signal rapport for noise $\mathrm{RZS}=13 \mathrm{~dB}$, after 100 iterations using:

a) ACS algorithm

b) ACSF algorithm

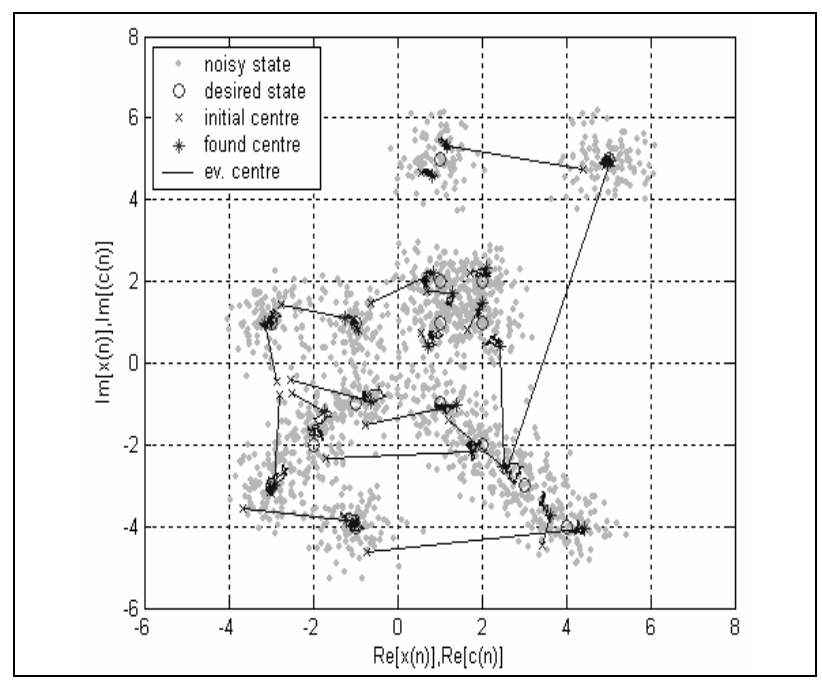

Fig. 5. Illustration of ACS algorithm

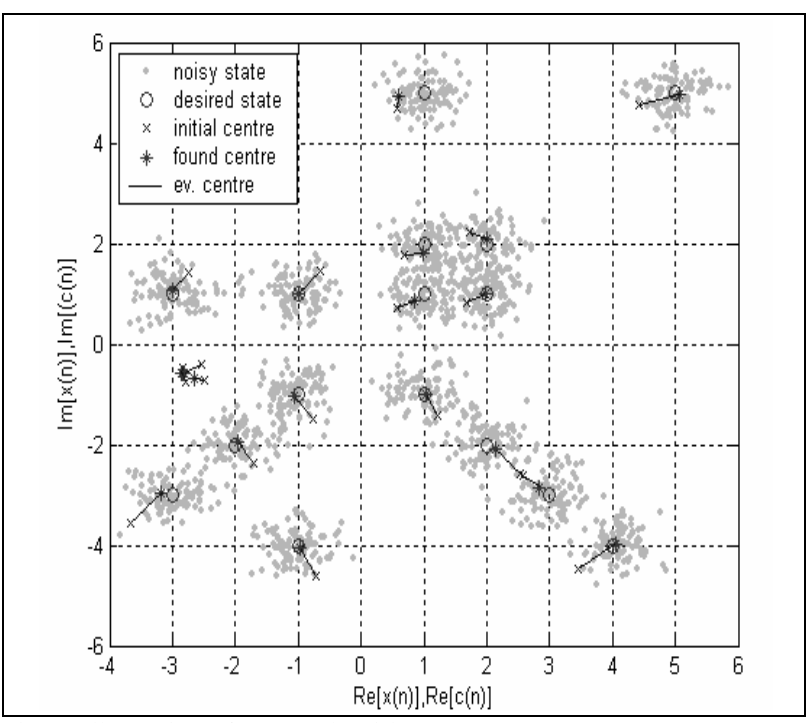

Fig. 6. Illustration of ACSF algorithm

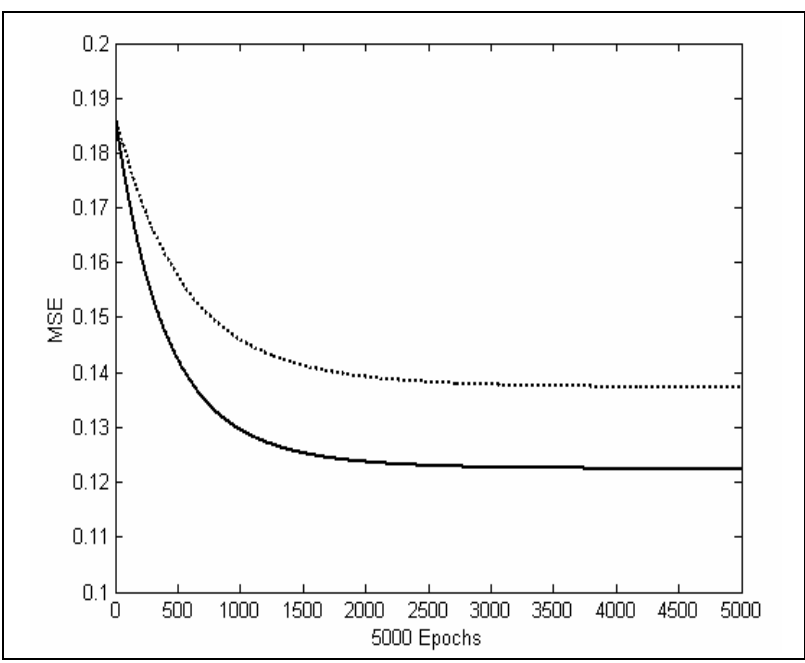

Fig. 7. Comparative evolution of the square mean error of equalizer RBFR during training, for a rapport $\mathrm{RSZ}=13 \mathrm{~dB}$ and order $m=1$ of filter LT (continuous line - ACSF algorithm, dotted line - ACPR algorithm)

\section{CONCLUSIONS}

The performances of RBFR equalizers are superior to those of linear equalizers and RNM implemented equalizers. Competitive algorithms eliminate the disadvantages of above mentioned RNM equalizers. Note that competitive algorithm with penalizing the rival that efficiently determine RBFR centers by gratifying the winner and penalizing the rival is simple, it generates regions of decision strongly nonlinear and has a rapid convergence. By comparing it with the ACSF algorithm, it has a much faster convergence and the determined centers have positions closer to the wanted ones. ACPR algorithm is adequate for the adaptive equalization of complex signals rapid variables in time, affected by linear and nonlinear distortions. The obtained performance, a square mean error of $10-2$ for a RSZ of $5 \mathrm{~dB}$ is similar to that of other neuronal equalizers with RBFR reported in literature, tested in the same conditions.

The adaptation of the positions of the centers of the radial basis functions does not necessarily provide the best results. The success of the moving centers depends on the 
application of interest. There are some applications for which adaptation of the centers of the RBFs has practical advantages. For example, it allows us to define the classes (input patterns) better and more accurately. The following chapter gives some applications of RBFNs in various fields [19].

\section{REFERENCES}

[1] C. Vertan ,M. Ciuc, M. Zamfir, "Prelucrarea si analiza imaginilor", Printech Publishing House Bucharest, 1999, ISBN 973- 9475-71

[2] A. G. Bors, I. Pitas, . Robust RBF Networks. In R. J. Howlett, L. C. Jain (eds.), Radial basis function neural networks: design and applications, pp. 125-153. Physica-Verlag, 2001

[3] S. Papadimitriou, S. Mavroudi, L. Vladutu and A. Bezerianos. Generalized Radial Basis Function Networks Trained with Instance Based Learning for Data Mining of Symbolic Data. Applied Intelligence, 16, pp. 223-234, 2002

[4] Radu DOGARU,Universality and emergent computation in cellular neural networks, World Scientific Series on Nonlinear Science, Series A - Vol. 43, ISBN: 978-981-238-102-6, Mar 2003

[5] D.Dumitrescu, K.Simion, "Reducing complexity of RBF neural networks by dynamic evolutionary clustering techniques", Proc. of $11^{\text {th }}$ Conference on Applied and IndustrialMathematics, 2003, pp. $83-89$

[6] I.C. Vizitiu, Networks and genetic algorithms. Theory and applications (2004)

[7] R. Dogaru, "A Hardware Oriented RBF Classifier with a Simple Constructive Training Algorithm Based on Support Vectors CSCS-16", 16th International Conference on control systems and computer science May, 2007, Politehnica University of Bucharest

[8] I.C.Vizitiu, D.Munteanu, "A genetic procedure for RBF neural network center selection", Proc. Of the 9th WSEAS International Conference NN'08,2008, pp. 37-40

[9] I.C.Vizitiu, F.Şerban, C.Molder, M.Stanciu,"Decision fusion method to improve the performances of multispectral ATR systems",Proc. of 1st WSEAS International Conference SENSIG'08, 2008, pp.40-45

[10] Training of RFB neural networks using a full-genetic approach Iulian-Constantin VIZITIU, Petrică CIOTÎRNAE, Teofil OROIAN, Adrian RADU Florin POPESCU, Cristian AVRAM ISSN: 1790-0832. 1015. Issue 8, Volume 7, WSEAS TRANSACTIONS on INFORMATION SCIENCE and APPLICATIONS August 2010

[11] D.K.Roy, L.K.Sharma, "Genetic k-means clustering algorithm for mixed numeric and categorical data sets", Journal of Artificial Intelligence\&Applications, vol.1, 2010, pp. 23-28

[12] I.C.Vizitiu, I.C.Rincu, I.Nicolaescu, "Optimal FPGA implementation of GARBF systems", Proc. of 12nd Conference OPTIM, 2010, pp.774-77

[13] I. Cha, S.A. Kassam, "Channel Equalization using Adaptive Complex Radial Basis Function Networks", IEEE J. Selected Areas in Communications, 13, 122-131, January 1995, Special Issue on Intelligent Signal Processing

[14] I. Cha, S.A. Kassam, "RBFN Restoration of Nonlinearly Degraded Images", IEEE Trans Image Processing, 5, June 1996, Special Issue on Nonlinear Image Processing.

[15] Y. Li, "Pilot-symbol-aided channel estimation for OFDM in wireless systems," IEEE Transactions on Vehicular Technology, vol. 49, no. 4, pp. 1207-1215, Jul. 2000

[16] S. Coleri, M. Ergen, A. Puri, and A. Bahai, "Channel estimation techniques based on pilot arrangement in OFDM systems," IEEE Transactions on Broadcasting, vol. 48, no. 3, pp. 223 - 229, Sept. 2002

[17] O. Edfors, M. Sandell, J.-J. van de Beek, S. Wilson, and P. Borjesson, "OFDM channel estimation by singular value decomposition," IEEE Transactions on Communications, vol. 46, no. 7, pp. 931-939, Jul. 1998

[18] S. K. P. Hoeher and P. Robertson, "Two-dimensional pilotsymbol-aided channel estimation by Wiener filtering," in Proceeding of IEEE Conference on Acoustics, Speech, and Signal Processing, 1997, pp. 1845-1848
[19] I. Cha and S. Kassam, "Channel equalization using adaptive complex radial basis function networks," IEEE Journal on Selected Areas in Communications, vol. 13, no. 1, p. Jan., Jan. 1995

[20] X. Zhou and X. Wang, "Channel estimation for OFDM systems using adaptive radial basis function networks," IEEE Transactions on Vehicular Technology, vol. 52, no. 1, pp. 48-99, Jan. 2003

[21] "DTV receiver performance test report - test report of multipath testing performed at ATTC," download available at http://www.attc.org/

[22] S. Chen , G. J. Gibson , C. F. N. Cowan , P. M. Grant, Adaptive equalization of finite non-linear channels using multilayer perceptions, Signal Processing, v.20 n.2, p.107-119, Jun. 1990

[23] S. Chen , G. J. Gibson , C. F. N. Cowan , P. M. Grant, Reconstruction of binary signals using an adaptive radial-basisfunction equalizer, Signal Processing, v.22 n.1, p.77-93, Jan. 1991

[24] Chen, S., Mulgrew, B. and Grant, P. M.: A clustering technique for digital communications channel equalization using radial basis function networks. In IEEE Transactions on Neural Networks, 4, (4) (1993), 570-579

[25] Kechriotis, G., Zervas, E. and Manolakos, E. S.: Using recurrent neural networks for adaptive communication channel equalization.IEEE Transactions on Neural Networks, 5, (2) (1994), 267-278

[26] Parisi, R., Claudio, E. D. D., Orlandi G. and Rao, B. D.: Fast adaptive digital equalization by recurrent neural networks, IEEE Transactions on Signal Processing, 45, (11) (1997), 2731-fb2739

[27] Kumar, P. C., Saratchandran, P. and Sundararajan, N.: Minimal radial basis function neural networks for nonlinear channel equalisation. IEEE Proceedings, Vision, Image and Signal Processing, 147 (5) (2000), 428-435

[28] Huang, G.-B., Chen, Y. Q. and Babri, H. A.: Classification ability of single hidden layer feedforward neural networks, IEEE Transactions on Neural Networks, 11, (3) (2000), 799- 801

[29] Huang, G.-B., Saratchandran, P. and Sundararajan, N.: An efficient sequential learning algorithm for Growing and Pruning RBF (GAP-RBF) networks, IEEE Transactions on Systems, Man and Cybernetics-Part B: Cybernetics, 34, (6), (2004), 2284-2292

[30] D.G. Khairnar, S.N. Merchant, U.B. Desai, Radar Signal Detection in Non-Gaussian Noise using RBF Neural Network, Journal of Computers, Vol. 3, No. 1, January 2008

[31] Z. Michalopoulou, L. Nolta and D. Alexandrou,: Performance evaluation of multilayer perceptrons in signal detection and classification, IEEE Transactions on Neural Networks, Vol.6, No.2 (1995)

[32] P. P. Gandhi and V. Ramamurti,: Neural networks for signal detection in non-Gaussian noise, IEEE Transactions on Signal Processing, Vol.45, No.11 (1997)

[33] V. Ramamurti, S.S. Rao, and P.P. Gandhi: Neural detectors for signals in non-Gaussian noise, In IEEE International Conference Acoustic, Speech, Signal Processing, Minneapolis, MN, 1993; Reprinted in Neural Networks: Theory, Technology, and Applications, P.K. Simpson, ED. Piscataway, NJ:IEEE (1996)

[34] L. Fa-Long and U. Rolf,: Applied Neural Networks for Signal Processing, Cambridge University Press, (1997)

[35] S. C. Chen and P. M. Grant,: Orthogonal least squares learning algorithm for radial basis function networks, IEEE Transactions on Neural Networks, Vol.2, No.2, (1991) 302-309

[36] Michael Turley,: Impulsive noise rejection in HF radar using a linear prediction technique, IEEE Radar,(2003) 358-362

[37] J. R. Barnum and E. E. Simpson,: Over-the-Horizon radar sensitivity enhancement by impulsive noise excision, IEEE Radar (1997) $252-256$

[38] 77. Yaron Minsky, Ari Trachtenberg and Richard Zippel. Set Reconciliation with Nearly Optimal Communications Complexity. In ISIT, 2001

[39] 78. F. Monrose, M.K. Reiter, Q. Li and S. Wetzel. Cryptographic key generation from voice. In IEEE Symp. on Security and Privacy, 200

[40] 79. N. Ratha, J. Connell and R. Bolle. Enhancing security and privacy in biometrics-based authentication systems. IBM Systems Journal, 40(3):614-634, 2001

[41] 80. Michael D.Garris and R.Michael McCabe. Fingerprint Minutiae From Latent and Matching Tenprint Images. NIST Special Database 27, 2000 